\title{
Pathological changes caused by Anoplocephala perfoliata in the equine ileocecal junction
}

\author{
S. Pavone • F. Veronesi $\cdot$ D. Piergili Fioretti • \\ M. T. Mandara
}

Published online: 12 May 2010

(C) Springer Science+Business Media B.V. 2010

\begin{abstract}
Gastrointestinal motility disorders represent a significant cause of morbidity and mortality in horses. Previously regarded as a non-pathogenic tapeworm, Anoplocephala perfoliata has been recently associated with equine colic. In this study, pathological changes related to $A$. perfoliata at the ileocecal junction were investigated in 31 slaughtered horses. Our results showed a significant relationship between parasitic burden and grading of histopathological lesions in both the mucosa and submucosa. Moreover, in infested horses, hypertrophy of the circular muscle layer was determined. Finally, an enteric nervous system (ENS) evaluation showed injury to intestinal nervous elements in horses with moderate to high parasitism. In summary, our results on the ENS support a correlation between colic and $A$. perfoliata infestion in the horse.
\end{abstract}

Keywords Equine Anoplocephala perfoliata Ileocecal junction .

Enteric nervous system $\cdot$ ENS

\section{Abbreviation \\ ENS Enteric nervous system}

\section{Introduction}

Intestinal colic is one of the most important causes of morbidity and mortality in horses. For this reason, several epidemiological studies have been performed to identify the potential risk factors of this condition. For many years, Anoplocephala perfoliata has been regarded as an incidental finding in the gut of horses, rarely associated with intestinal colic syndrome. However, since the 1980s, an increasing prevalence of case reports describing a close association between specific causes of equine colic, such as impaction (Proudman et al. 1998), intestinal rupture (Beroza et al. 1983), intussusception (Barclay et al. 1982; Edwards 1986; Owen et al. 1989), volvulus, and large intestine obstructions (Beroza et al.

S. Pavone $(\bowtie) \cdot$ F. Veronesi $\cdot$ D. Piergili Fioretti $\cdot$ M. T. Mandara Dipartimento di Scienze Biopatologiche ed Igiene delle Produzioni Animali e Alimentari, Facoltà di Medicina Veterinaria, Università degli Studi di Perugia,

Via S. Costanzo 4, 06126 Perugia, Italy

e-mail: pavone.silvia@libero.it 
1983; Carmel 1988; Slocombe 1979) and severe A. perfoliata infestations has increased the interest in the pathological effects of this parasite. On the other hand, in the absence of apparent organic causes, a key factor in colic intestinal syndrome has been attributed to gut dismotility caused by myenteric and submucosal ganglionitis (Burns et al. 1990). However, no sufficient studies on Enteric Nervous System (ENS) lesions supporting these hypotheses have been reported in the literature.

Since the ENS plays a key role in intestinal motor activity, the aim of this study is to investigate the pathological changes of ENS components that occur in association with A. perfoliata infestation/burden of the ileocecal junction, the favourite attachment site of this parasite.

\section{Materials and methods}

Thirty-one mixed-breed horses, ranging from 15 months to 13 years of age and regularly sent to slaughterhouses, were randomly selected for this study. They included 11 parasite-free animals (control group) and 20 horses affected by spontaneous $A$. perfoliata infection. In all horses, the ileocecal junction was collected within 30 minutes of death. Subsequently, intestinal samples were rinsed in running water and placed immediately in 10\% neutral-buffered formalin. Meanwhile, adult tapeworms attached to the ileocecal junction were directly counted, recorded, rinsed in running water, and preserved in a $10 \%$ formalin solution. The collected tapeworms were put in lactophenol solution and then mounted on slides to be identified using morphological parameters (Euzeby 1981). Based on the number of tapeworms recorded, the selected 31 horses were divided in four groups: group "N," with 0 tapeworms; group "A," with 1 to 20 tapeworms; group "B," with tapeworms ranging from 20 to 100; and group "C," with more than 100 tapeworms.

From each sample, 5- $\mu \mathrm{m}$ thick paraffin-embedded sections were stained with hematoxylin-eosin and cresyl violet. Histological investigation was performed to evaluate the following parameters: lymphomonocytic and eosinophilic inflammatory infiltration in the mucosa/submucosa, atrophy/fusion of intestinal villi, erosive/ulcerative lesions, degenerative changes of goblet cells, and intestinal lymphatic follicle hyperplasia. A score was individually attributed to each selected parameter: 0 , absence of lesions; 1 , mild lesions; 2, moderate lesions; and 3, marked lesions. Consequently, based on the sum total of the recorded scores, the mucosal and submucosal damage was graded as follows: 0 (no changes) with 0-2 points; I (mild changes) with 3-7 points, II (moderate changes) with $8-12$ points, and III (marked changes) with $13-15$ points.

At the ileocecal junction, the thicknesses of circular and longitudinal muscle layers were quantified by image analysis (NIS-Elements Br-2) to identify hypertrophic conditions. For each sample, ENS histopathological investigation was assessed by morphometric evaluation to assess the average linear density for myenteric ganglia, neuronal cell number, and the average percentage of chromatolytic/necrotic neuronal cells in three 3-cm serial sections.

A general linear model (GLM) was used to verify a significant correlation between the parasitic burden and histopathological grading of the mucosal and submucosal lesions. A one-way analysis of variance (ANOVA) followed by Tukey's Multiple Comparison test were used to detect significant differences between the number of myenteric ganglia, the number of neuronal cells, the percentage of chromatolytic/necrotic neurons, the thicknesses of muscle layers, and the group identified based on the number of tapeworms recorded. Five percent was the assumed significance level. Statistical analysis was carried out using R 
2.7 version software (GNU GENERAL PUBLIC LICENSE, Free Software Foundation, Inc., Boston, USA).

\section{Results}

Based on the number of recorded tapeworms, 11 horses were included in group "N" (0 parasites = control group), 15 in group "B" (20-100 tapeworms), and 5 in group "C" (> 100 tapeworms). No spontaneously affected horses harboured less than 20 tapeworms to be included in group "A." At gross examination, the parasite-free horses (group $\mathrm{N}$ ) showed normal pinkish bright mucosa, while the horses of groups B and C showed erosive-ulcerative lesions, sometimes covered by diphteric membranes. These lesions appeared more severe in group $\mathrm{C}$ than in group $\mathrm{B}$.

Histological examination confirmed the presence of large areas of ulceration with cellular debris, fibrin, erythrocytes, neutrophils, and bacterial colonies in the horses of B and $\mathrm{C}$ groups. The most affected areas showed goblet cell loss or a marked degeneration with severe vacuolization, and atrophy and fusion of intestinal villi. Moreover, histopathological examination allowed the assessment of the presence, type, and extent of inflammatory infiltration. The horses of group $\mathrm{N}$ showed a mild, mainly eosinophilic infiltration restricted to the lamina propria. On the contrary, the horses of groups $\mathrm{B}$ and $\mathrm{C}$ showed a severe and deep mixed inflammation that extended to the muscle layers. The lymphocytes appeared to be more numerous than eosinophils in group C. Marked edema associated with lymphatic vessel ectasia was also detected in the submucosa. Histopathological evaluation showed severe submucosal lymph follicle hyperplasia in the horses of group B.

Based on the reported histopathological data, the sum total of recorded scores allowed the identification of grade 0 lesions in all horses of group $\mathrm{N}$ and grade II lesions (moderate) in the horses of the group B (except for one showing a grade III lesion); the horses of group $\mathrm{C}$ were affected by grade III lesions (marked). Statistical analysis confirmed a significant relationship between parasitic burden and histopathological grading of lesions $(P \leq 0.05)$.

Muscle layer evaluation revealed an increased circular muscle layer thickness (hypertrophy) in the horses of group $\mathrm{B}$ and $\mathrm{C}$ compared to group $\mathrm{N}(P \leq 0.05)$; this finding was not statistically related to the parasitic burden $(P>0.05)$.

Morphological and morphometrical evaluation of ENS showed a significant decrease in myenteric plexi and neuronal cells, and an increase in chromatolysis and necrosis in parasitized horses compared to parasite-free animals. In three horses of group $\mathrm{C}$, lymphocytic-plasmacytic periganglionitis and ganglionitis were also observed. Statistical analysis showed a significant damage of ENS in the horses of groups B and C compared to group $\mathrm{N}$, but this finding was not related to parasitic burden $(P>0.05)$.

\section{Discussion}

The results collected at the ileocecal junction of the selected horses confirmed a close relationship between parasitic burden and grading of mucosal and submucosal histopathological lesions. Moreover, the results showed significant damage of the ENS in all horses with a moderate parasitic burden (20-100 tapeworms, group B) and with high parasitism ( $>100$ tapeworms, group C). Although in our study the parasitic burden did not appear statistically correlated to ENS damage, our results might support a correlation between colic 
and A. perfoliata infestion in the horse; in this case, tapeworms may represent a cause of ENS failure and, consequently, of intestinal motility impairment. Finally, the ENS lesions we observed in horses with a moderate parasitic burden suggest the use of well-timed diagnostic tests and orderly preventive deworming programs also for moderate infestations to prevent or minimize the risk of colic caused by A. perfoliata.

\section{References}

Barclay WPT, Philips J, Foerner J (1982) Intussusception associated with Anoplocephala perfoliata infection in 5 horses. J Am Vet Med Assoc 180:752-53

Beroza GA, Williams R, Marcus LP, Mille P (1983) Prevalence of tapeworm infection and associated large bowel disease in horses. Proceedings of Equine Colic Research Symposium 2:711-724

Burns GN, Karcher LF, Cummings JF (1990) Equine myenteric ganglionitis: A case of chronic intestinal pseudo-obstruction. Cornell Vet 80:53-63

Carmel DK (1988) Tapeworm infection in horses. J Equine Vet Sci 8:343.

Edwards, G.B., 1986. Surgical management of intussusception in the horse. Equine Vet J 18:313

Euzeby J (1981) Diagnostic experimental des animales. In: Informations techniques des Services Veterinaires. Ministere de l'Agricolture, Paris, France, p 174

Owen,RA, Jagger DW, Quan-Taylor R (1989) Caecal intussusceptions in horses and the significance of Anoplocephala perfoliata. Vet Rec 124:34-37

Proudman CJ, French NP, Trees AJ (1998) Tapeworm infection is a significant risk factor for spasmodic colic and ileal impaction colic in the horse. Equine Veterinary Journal, 30, 194-199

Slocombe JOD 1979. Prevalence and treatment of tapeworms in horses. Can Vet J 20:136-140 